\title{
Behavior of topological defects of optical indicatrix orientation in cubic single crystals under conically distributed electric field. 1. The electric field and the optical beam parallel to the three-fold symmetry axis
}

\author{
Vasylkiv Yu., Kryvyy T., Skab I. and Vlokh R. \\ Vlokh Institute of Physical Optics, 23 Dragomanov Street, 79005 Lviv, Ukraine, \\ vlokh@ifo.lviv.ua
}

Received: 09.10.2014

\begin{abstract}
We have studied the behaviour of topological defects (TDs) of optical indicatrix orientation under the conditions when the electrooptic Pockels and Kerr nonlinearities coexist in cubic crystals and the axes of both conically shaped electric field and light beam are parallel to the crystallographic direction [111]. We have found that the electric voltage leads to a birth of three pairs of TDs. They are characterized by half-integer magnitudes of their strength and the opposite signs of that strength within the pairs. The above pairs of TDs annihilate with decreasing voltage. We have also shown that the total charge of relevant optical vortices is not conserved under topological reactions.
\end{abstract}

Keywords: Pockels effect, Kerr effect, topological defects, optical indicatrix, optical vortices

PACS: $78.20 . J q, 42.50 . T x$

UDC: $535.5+537.228 .3+515.1$

\section{Introduction}

Topological defects (TDs) of optical indicatrix orientation produce polarization singularities in an optical beam propagating through anisotropic inhomogeneous media and result in appearance of optical vortices (see, e.g., Ref. [1]). As an example, TDs associated with the director and produced technologically in liquid crystals are successfully used for generating optical vortices [2]. Such devices are commonly called as q-plates [3]. They can be applied in a number of novel branches of optical signal processing, e.g. in optical cryptography, information processing, quantum teleportation, etc. [4-8]. Though the TDs in liquid crystals are quite easily created, the materials mentioned above are often temperature unstable and the range of their applications is somewhat restricted due to their excessive pliability to external fields.

Recently we have shown [9-14] that the TDs of optical indicatrix orientation can also be induced by spatially inhomogeneous external fields applied to single crystals. For instance, TDs with the strengths $+1 / 2$ or $-1 / 2$ can be induced under crystal bending or torsion, respectively via a piezooptic effect [9-11]. The defects with the strength $-1 / 2$ are produced via an electrooptic Pockels effect whenever a conically distributed electric field is applied [12, 13]. Similarly, the TDs with the strength equal to +1 appear as a result of a quadratic electrooptic (Kerr) effect under a conically shaped electric field [14]. Creation of the TDs with different strengths via the Pockels and Kerr effects has stipulated the analysis of behaviours of those defects in an 'intermediate' regime occurring under conditions when both of the effects coexist [15]. In particular, we have found [15] that the change, from $-\frac{1}{2}$ to +1 , in the strength of the central 
TD cannot be observed under increasing electric field. Instead, we have revealed a 'crossover' regime in the process of creation of the TDs. It is an intermediate mode appearing between the conditions under which one of the competing electrooptic nonlinearities, Pockels or Kerr ones, prevail. The crossover arises when the electric voltage increases such that the Kerr effect manifests itself in a notable manner. Instead of the TD with the unit strength, which would have produced a doubly charged OV, four different TDs then appear, with their strengths equal to $\pm 1 / 2$. Further increase in the electric field shifts the additional lateral TDs towards the central one [15]. Besides, the study [15] has specified the point symmetry groups for which both the Pockels and Kerr effects coexist as the groups $\overline{4} 3 \mathrm{~m}, 23, \overline{6} \mathrm{~m} 2, \overline{6}, \overline{4}, 3 \mathrm{~m}, 32$, and 3 .

In spite of some progress reached in the work [15] and concerned with studying electrooptically induced TDs in solid-state crystals, only the TDs appearing in the crystals of the point group $\overline{6} \mathrm{~m} 2$ have been analyzed there. This has been done thanks to a relative simplicity of theoretical relations that describe the field-induced phase difference and optical indicatrix orientation for this symmetry group. The other groups do not reveal such encouraging circumstances. For the case of cubic symmetry groups ( $\overline{4} 3 \mathrm{~m}$ and 23 ), of which electrooptic tensors include the smallest number of independent components, it has been concluded [15] that, at least under a conical field with the cone axis parallel to the principal crystallographic axes, the crossover regime cannot appear because the Pockels effect produces no TD of optical indicatrix orientation in the centre of the beam cross-section. The appearance of TDs with the strength 1 due to the Kerr effect in the cubic crystals has not been also analyzed in Ref. [15]. Here we are to notice that there exists an additional possibility for creating the TDs in the cubic crystals: they can be produced in the centre of the beam crosssection when the conical field is applied along the direction [111]. This interaction geometry can lead to the crossover regime because electrooptically induced birefringence should not appear when the electric field is applied along the three-fold axes and the light propagates along the same directions. The aim of the present work is to analyze in detail this particular experimental geometry.

\section{Methods of analysis}

For simulating a so-called 'effective' angle $\zeta_{k l}^{e f}$ of optical indicatrix orientation and an 'effective' phase difference $\Delta \Gamma_{k l}^{e f}$ for each elementary ray belonging to a wide Gaussian beam, one can use a well-known Jones matrix approach, by dividing a sample by, e.g., $S_{\max }=100$ homogeneous layers perpendicular to the crystallographic axis [111]. In its turn, each of the layers is divided by $k \times l$ homogeneous elementary cells in the $X^{\prime} Y^{\prime}$ plane. Here $Y^{\prime}$ and $X^{\prime}$ axes are parallel to the crystallographic directions $[\overline{1} 10]$ and $[11 \overline{2}]$, respectively.

The detailed description of application of the Jones matrices for simulation of the optical indicatrix orientation and the phase difference in crystals under conical electric field has been presented in Refs. [12-15]. In particular, in the recent work [15] phenomenological relations for the birefringence and the optical indicatrix orientation induced by a conically shaped electric field inside the homogeneous layers have been derived for all of the aforementioned symmetry groups. When the conical field axis is aligned with the [111] axis and the light beam propagates along the same direction, the birefringence and the optical indicatrix orientation for the crystals belonging to the point group $\overline{4} 3 \mathrm{~m}$ read respectively as 


$$
\begin{aligned}
& \Delta n_{X^{\prime} Y^{\prime}}=-\frac{n^{3}}{2} \sqrt{\begin{array}{l}
\left.\left(E_{1}-E_{2}\right)\left(2 r_{11}^{\prime}+\left(R_{11}^{\prime}-R_{12}^{\prime}\right)\left(E_{1}+E_{2}\right)-2 R_{14}^{\prime} E_{3}\right)\right)^{2} \\
+4\left(\left(E_{1}+E_{2}\right)\left(R_{14}^{\prime} E_{3}-r_{11}^{\prime}\right)+R_{66}^{\prime} E_{1} E_{2}\right)^{2}
\end{array}} \\
& \tan 2 \zeta_{Z^{\prime}}=\frac{2\left(\left(E_{1}+E_{2}\right)\left(R_{14}^{\prime} E_{3}-r_{1}^{\prime}\right)+R_{66}^{\prime} E_{1} E_{2}\right)}{\left(E_{1}-E_{2}\right)\left(2 r_{11}^{\prime}+\left(R_{11}^{\prime}-R_{12}^{\prime}\right)\left(E_{1}+E_{2}\right)-2 R_{14}^{\prime} E_{3}\right)} .
\end{aligned}
$$

Here $n$ is the refractive index, the terms $E_{1}=k X, E_{2}=k Y$ and $E_{3}=k Z$ denote the electric field components $\quad\left(k=U Z / d\left(X^{2}+Y^{2}+Z^{2}\right)\right), \quad$ and $\quad r_{11}^{\prime}=-\frac{1}{\sqrt{3}} r_{41}, \quad R_{11}^{\prime}=\frac{1}{2}\left(R_{11}+R_{44}+R_{12}\right)$, $R_{12}^{\prime}=\frac{1}{6}\left(R_{11}-R_{44}\right)+\frac{5}{6} R_{12}, \quad R_{14}^{\prime}=\frac{1}{3}\left(R_{11}-R_{44}-R_{12}\right) \quad$ and $\quad R_{66}^{\prime}=\frac{1}{3}\left(R_{11}+2 R_{44}-R_{12}\right) \quad$ are the electrooptic components. Namely, $R_{\lambda \mu}^{\prime}$ and $r_{\lambda k}^{\prime}$ are respectively the Kerr and Pockels tensor components rewritten in the coordinate system $X^{\prime} Y^{\prime} Z^{\prime}$, of which $Z^{\prime}$ axis is parallel to [111] and $Y^{\prime}$ and $X^{\prime}$ to $[\overline{1} 10]$ and $[11 \overline{2}]$, respectively. A standard matrix notation is used for the tensor components, i.e. the Kerr coefficients are represented as $R_{\lambda \mu}=R_{i j k l}$ for $i j \leftrightarrow \lambda=1, \ldots 6 ; k l \leftrightarrow \mu=1,2,3$ and $R_{\lambda \mu}=2 R_{i j k l}$ for $i j \leftrightarrow \lambda=1, \ldots 6 ; k l \leftrightarrow \mu=4,5,6$. Similarly, the Pockels coefficients are written as $r_{\lambda k}=r_{i j k}$.

In the spherical coordinate system defined by $X=\rho \sin \Theta \cos \varphi, Y=\rho \sin \Theta \sin \varphi$ and $Z=\rho \cos \Theta$, we obtain $E_{1}=U \tan \Theta \cos \varphi / d\left(1+\tan ^{2} \Theta\right), E_{2}=U \tan \Theta \sin \varphi / d\left(1+\tan ^{2} \Theta\right)$ and $E_{3}=U / d\left(1+\tan ^{2} \Theta\right)$, where $U$ denotes the electric voltage applied, $d$ the thickness of crystalline plate, and $E_{0}=U / d$. It is evident that the $E_{1}$ and $E_{2}$ components are equal to zero if $\Theta=0$ (the case of a homogeneous field, with field lines parallel to the $Z$ axis). These components increase with increasing $\Theta$ and decreasing $d$ (see Fig. 1).

Under the same conditions as above, the corresponding relations for the crystals belonging to the point group 23 become as follows:

$$
\begin{gathered}
\Delta n_{X^{\prime} Y^{\prime}}=-\frac{n^{3}}{2} \sqrt{\begin{array}{l}
\left.\left(E_{1}-E_{2}\right)\left(2 r_{11}^{\prime}+\left(R_{11}^{\prime}-R_{12}^{\prime}\right)\left(E_{1}+E_{2}\right)\right)+2 E_{3}\left(R_{14}^{\prime} E_{2}+R_{15}^{\prime} E_{1}\right)+2 R_{16}^{\prime} E_{1} E_{2}\right)^{2}+ \\
+4\left(\left(E_{1}+E_{2}\right)\left(r_{11}^{\prime}+\frac{1}{2} R_{16}^{\prime}\left(E_{1}-E_{2}\right)\right)+E_{3}\left(R_{15}^{\prime} E_{2}-R_{14}^{\prime} E_{1}\right)-R_{66}^{\prime} E_{1} E_{2}\right)^{2}
\end{array}} \\
\tan 2 \zeta_{Z^{\prime}}=-\frac{\left(E_{1}+E_{2}\right)\left(2 r_{11}^{\prime}+R_{16}^{\prime}\left(E_{1}-E_{2}\right)\right)+2 E_{3}\left(R_{15}^{\prime} E_{2}-R_{14}^{\prime} E_{1}\right)-2 R_{66}^{\prime} E_{1} E_{2}}{\left(E_{1}-E_{2}\right)\left(2 r_{11}^{\prime}+\left(R_{11}^{\prime}-R_{12}^{\prime}\right)\left(E_{1}+E_{2}\right)\right)+2 E_{3}\left(R_{14}^{\prime} E_{2}+R_{15}^{\prime} E_{1}\right)+2 R_{16}^{\prime} E_{1} E_{2}} .
\end{gathered}
$$

Here $\quad r_{11}^{\prime}=-\frac{1}{\sqrt{3}} r_{41}, \quad R_{11}^{\prime}=\frac{1}{2}\left(R_{11}+R_{44}\right)+\frac{1}{4}\left(R_{12}+R_{21}\right)$,

$$
\begin{aligned}
& R_{12}^{\prime}=\frac{1}{6}\left(R_{11}-R_{44}\right)+\frac{5}{12}\left(R_{12}+R_{21}\right), R_{14}^{\prime}=\frac{1}{3}\left(R_{11}-R_{44}\right)-\frac{1+\sqrt{3}}{6} R_{12}-\frac{1-\sqrt{3}}{6} R_{21}, \\
& R_{15}^{\prime}=-\frac{1}{3}\left(R_{11}-R_{44}\right)+\frac{1-\sqrt{3}}{6} R_{12}+\frac{1+\sqrt{3}}{6} R_{21}, R_{16}^{\prime}=-\frac{\sqrt{3}}{6}\left(R_{12}-R_{21}\right), \text { and } \\
& R_{66}^{\prime}=\frac{1}{6}\left(2 R_{11}+4 R_{44}-R_{12}-R_{21}\right) .
\end{aligned}
$$




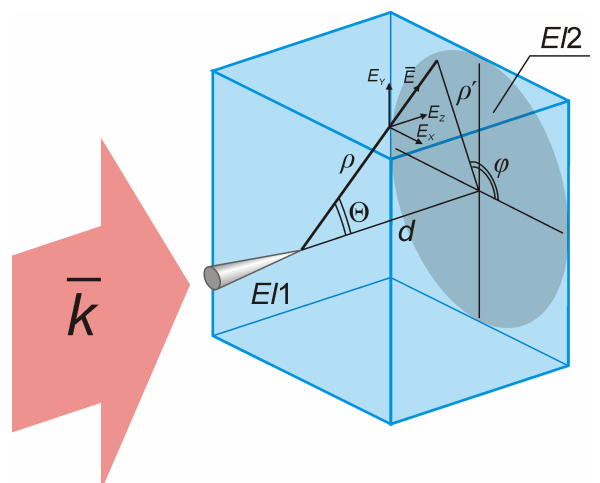

Fig. 1. Schematic representation of a crystalline plate with circularly shaped electrodes $e_{1}$ and $e_{2}$, and a conical spatial distribution of electric field produced by these electrodes.

When a sample with a TD of optical indicatrix orientation is placed in between crossed circular polarizers, one can observe a doughnut-mode intensity distribution peculiar to the optical vortices. Namely, the amplitude $\mathcal{E}^{\text {out }}(X, Y)$ of the outgoing light wave is described by

$$
\mathcal{E}^{\text {out }}(X, Y)=\mathcal{E}_{0} \cos \frac{\Delta \Gamma_{k l}^{e f}}{2}\left[\begin{array}{c}
1 \\
\pm i
\end{array}\right]+i \mathcal{E}_{0} \sin \frac{\Delta \Gamma_{k l}^{e f}}{2} e^{ \pm i 2 p \varphi \pm i 2 \zeta_{0}}\left[\begin{array}{c}
1 \\
\mp i
\end{array}\right],
$$

where $\Delta \Gamma_{k l}^{e f}$ denotes the phase difference, $l= \pm 2 p$ the topological charge of the optical vortex, $p$ the strength of the TD, $\zeta_{0}$ the angle of optical indicatrix orientation at $\varphi=0$, and $\mathcal{E}_{0}$ the amplitude of the incident light wave (see, e.g., Ref. [16]). The second term in the r. h. s. of Eq. (1) describes a helical mode (the charge of the optical vortex being equal to 1 at $p= \pm 1 / 2$ or 2 at $p= \pm 1$, while the first term describes the incident wave with no dislocation of its wave front.

This term vanishes at $\Delta \Gamma_{k l}^{e f}=\pi$.

\section{Results and discussion}

When performing our simulations, we use the following parameters of the crystal belonging to the symmetry group $\overline{4} 3 \mathrm{~m}: d=5 \mathrm{~mm}, n=1.7, \quad r_{11}^{\prime}=2 \times 10^{-12} \mathrm{~m} / \mathrm{V}, \quad R_{11}^{\prime}=1.75 \times 10^{-18} \mathrm{~m}^{2} / \mathrm{V}^{2}$, $R_{12}^{\prime}=0.583 \times 10^{-18} \mathrm{~m}^{2} / \mathrm{V}^{2}, \quad R_{14}^{\prime}=0.167 \times 10^{-18} \mathrm{~m}^{2} / \mathrm{V}^{2}$, and $R_{66}^{\prime}=1.167 \times 10^{-18} \mathrm{~m}^{2} / \mathrm{V}^{2}$. The diameter of the incident beam is taken to be equal to $\rho=5 \mathrm{~mm}$.

Let us analyze the behaviour of TDs in the crystal belonging to the point group $\overline{4} 3 \mathrm{~m}$, which is observed with increasing electric voltage. When the voltage is equal to $1 \mathrm{kV}$, only one TD of optical indicatrix orientation (referred to as $\mathrm{TD}_{0}$ ) appears in the beam centre (see Fig. 2a). The strength of this TD is equal to $-1 / 2$. The induced effective phase difference is equal to zero in the centre of the beam cross-section (see Fig.2 b), so that we have a singly charged optical vortex $\mathrm{OV}_{0}$ (see Fig. 2c). This optical vortex is almost canonical, since the dependence of the effective angle of optical indicatrix rotation on the tracing angle is close to linear (see Fig. 3).

The dependences of the coordinates of these two defects on the electric voltage are displayed in Fig. 5a. It is seen from Fig. 5a that, under the voltage $32.67 \mathrm{kV}$, those defects were born with the strengths $+1 / 2\left(\mathrm{TD}_{1}\right)$ and $-1 / 2\left(\mathrm{TD}_{1}{ }_{1}\right)$. They drift apart with increasing voltage: $\mathrm{TD}_{1}$ moves towards the centre of the beam cross-section, while $\mathrm{TD}^{*}{ }_{1}$ moves out of the beam aperture. To be more specific, $\mathrm{TD}^{*}{ }_{1}$ leaves the visible beam cross-section at $40 \mathrm{kV}$.

The rest of the solutions for the coordinates of $\mathrm{TD}_{2}, \mathrm{TD}_{2}, \mathrm{TD}_{3}$ and $\mathrm{TD}_{3}$ can be obtained only numerically. The behaviours of these defects with increasing field are similar to those of $\mathrm{TD}_{1}$ and TD* ${ }_{1}$ (see Fig. $5 \mathrm{~b}$ and Fig. $5 \mathrm{c}$ ). As the voltage increases up to $80 \mathrm{kV}, \mathrm{TD}_{1}, \mathrm{TD}_{2}$ and $\mathrm{TD}_{3}$ are 

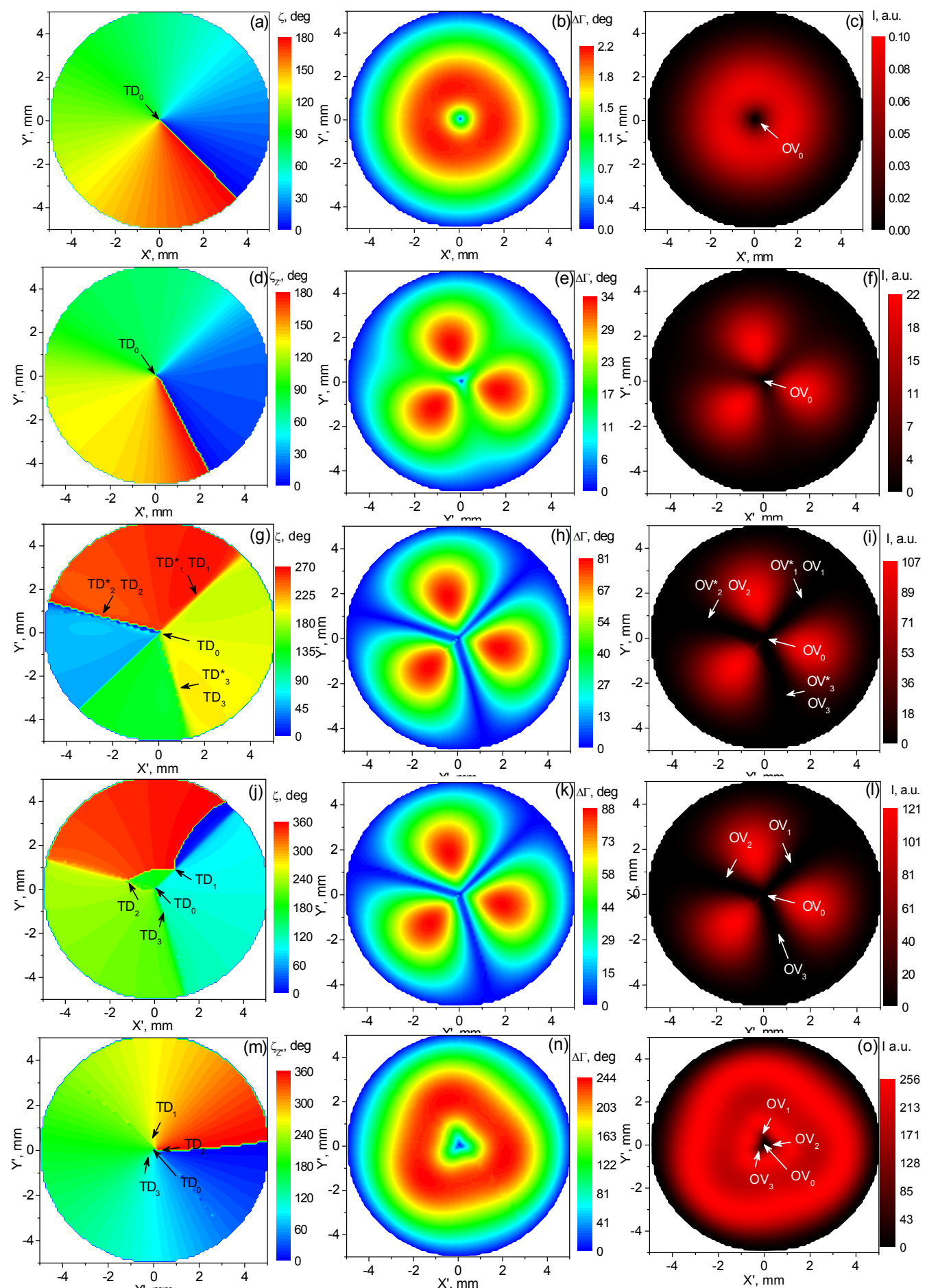

Fig. 2. Simulated spatial distributions for optical indicatrix rotation angle $(a, d, g, j, m)$, phase difference $(b, e, h, k, n)$ and light intensity behind the circular analyzer (c, f, i, l, o). The crystal belongs to the point group $\overline{4} 3 \mathrm{~m}$, and the axes of the conical external field and the light beam are both parallel to [111]. The electric voltages are equal to $1(\mathrm{a}-\mathrm{c}), 15(\mathrm{~d}-\mathrm{f}), 32.67(\mathrm{~g}-\mathrm{i}), 35(\mathrm{j}-\mathrm{l})$, and $80 \mathrm{kV}(\mathrm{m}-\mathrm{o})$.

located so close to $\mathrm{TD}_{0}$ that they cannot be resolved visually (see Fig. $2 \mathrm{~m}$ ). Nonetheless, the spatial distribution of the phase difference and the light intensity (see Fig. 2n, o) manifest peculiar 
triangular shapes. This means that the lateral defects approach the centre of the beam cross-section though they cannot reach it in the presence of the Pockels effect. Only when the Pockels coefficients tend to zero, it becomes possible to observe annihilation of one of the lateral defects with the central one and summing of the strength of the two other lateral defects, leading to a defect with the strength equal to +1 . The topological reaction describing this process may be obtained using a conservation law for the strengths of TDs, which represent quantum entities: $p_{\mathrm{TD}_{1}}+p_{\mathrm{TD}_{2}}+p_{\mathrm{TD}_{3}}+p_{\mathrm{TD}_{0}}=1 / 2+1 / 2+1 / 2-1 / 2=1$.
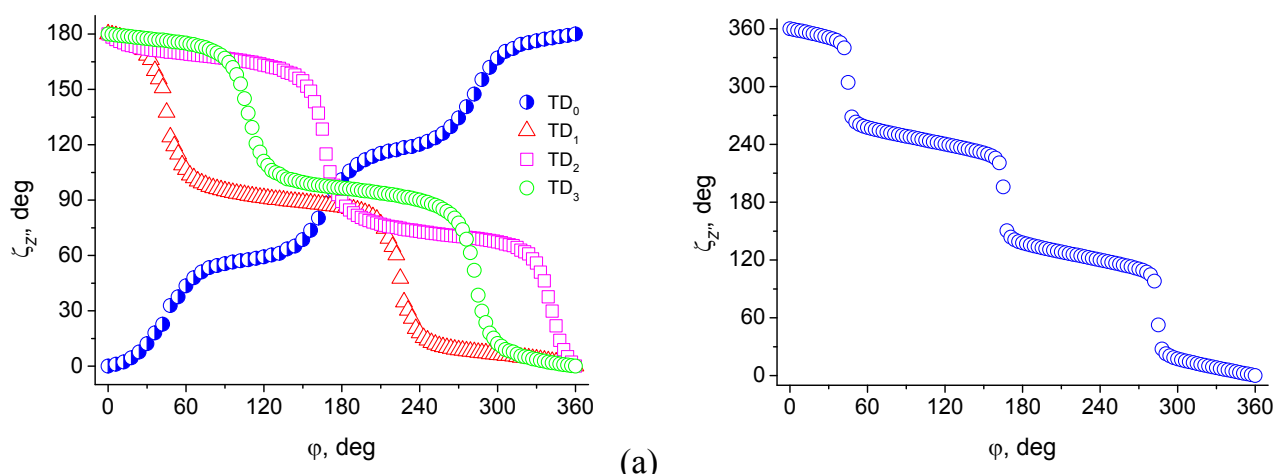

(a)

Fig. 3. Dependence of optical indicatrix rotation angle on the tracing angle at $U=35 \mathrm{kV}$ : a - at the radius $\rho=0.5 \mathrm{~mm}$ around the central and lateral TDs; $\mathrm{b}-$ at the radius $\rho=4.5 \mathrm{~mm}$ around the central TD.

When the voltage increases up to $15 \mathrm{kV}$, the dependence of the effective angle of optical indicatrix rotation on the tracing angle becomes nonlinear (see Fig. 3). Despite this fact, a single $\mathrm{TD}$ of optical indicatrix orientation $\left(\mathrm{TD}_{0}\right)$ with the strength equal to $-1 / 2$ is still observed in the beam centre (see Fig. 2d). This TD is surrounded by three maximums of the effective phase difference (see Fig. 2e), i.e. a singly charged optical vortex appears with inhomogeneous (triangular) spatial distribution of intensity of the ring of a doughnut mode (see Fig. 2f). At the voltage $32.67 \mathrm{kV}$, additional lateral TDs appear, which form a triangle (see Fig. $2 \mathrm{~g}$ ). When the voltage increases above $32.67 \mathrm{kV}$, six additional TDs of optical indicatrix orientation are observed $\left(\mathrm{TD}_{1}, \mathrm{TD}_{1}{ }_{1}, \mathrm{TD}_{2}, \mathrm{TD}_{2}, \mathrm{TD}_{3}\right.$ and $\mathrm{TD}_{3}{ }_{3}$ - see Fig. $2 \mathrm{j}, \mathrm{m}$ and Fig. 4). The strengths of these defects in the corresponding pairs are equal to $+1 / 2$ and $-1 / 2$. The effective phase difference is equal to zero in the places where they are located (see Fig. 2k). The coordinates of these TDs can be obtained by solving the system of equations which follows from Eq. (2):

$$
\left\{\begin{array}{l}
\left(E_{1}+E_{2}\right)\left(R_{14}^{\prime} E_{3}-r_{11}^{\prime}\right)+R_{66}^{\prime} E_{1} E_{2}=0 \\
\left(E_{1}-E_{2}\right)\left(2 r_{11}^{\prime}+\left(R_{11}^{\prime}-R_{12}^{\prime}\right)\left(E_{1}+E_{2}\right)-2 R_{14}^{\prime} E_{3}\right)=0
\end{array} .\right.
$$

One of the solutions is trivial: the system of Eqs. (6) is satisfied whenever $X_{0}=Y_{0}=0$. Obviously, these coordinates refer to the central defect $\mathrm{TD}_{0}$. The other two analytical solutions are

$$
X_{1,1^{*}}=Y_{1,1^{*}}, X_{1,1^{*}}=\frac{1}{8 r_{11}^{\prime} d}\left(R_{66}^{\prime} U Z \pm \sqrt{R_{66}^{\prime 2} U^{2} Z^{2}-32 r_{11}^{\prime} d\left(r_{11}^{\prime} d Z^{2}-R_{14}^{\prime} U Z^{2}\right)}\right) \text {. }
$$

When the voltage decreases, the process of interaction among the defects becomes reversible. Annihilation of $\mathrm{TD}_{1}$ with $\mathrm{TD}_{1}{ }_{1}, \mathrm{TD}_{2}$ with $\mathrm{TD}_{2}{ }_{2}$ and $\mathrm{TD}_{3}$ with $\mathrm{TD}_{3}{ }_{3}$ can be observed when the voltage decreases down to $32.67 \mathrm{kV}$. Obviously, since the strength of defects is conserved (e.g., for $\mathrm{TD}_{1}$ and $\mathrm{TD}^{*}$ ), we have $p_{\mathrm{TD}_{1}}+p_{\mathrm{TD}_{1}^{*}}=1 / 2-1 / 2=0$. The defect $\mathrm{TD}_{0}$ can disappear only if the conical electric field is switched off. 


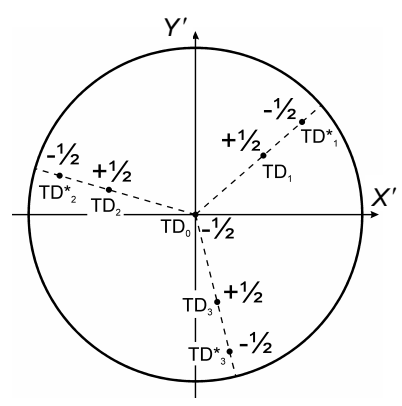

Fig. 4. Scheme of TDs of optical indicatrix orientation appearing in the crystal belonging to the point symmetry group $\overline{4} 3 \mathrm{~m}$ under application of conical electric field with the cone axis parallel to [111].
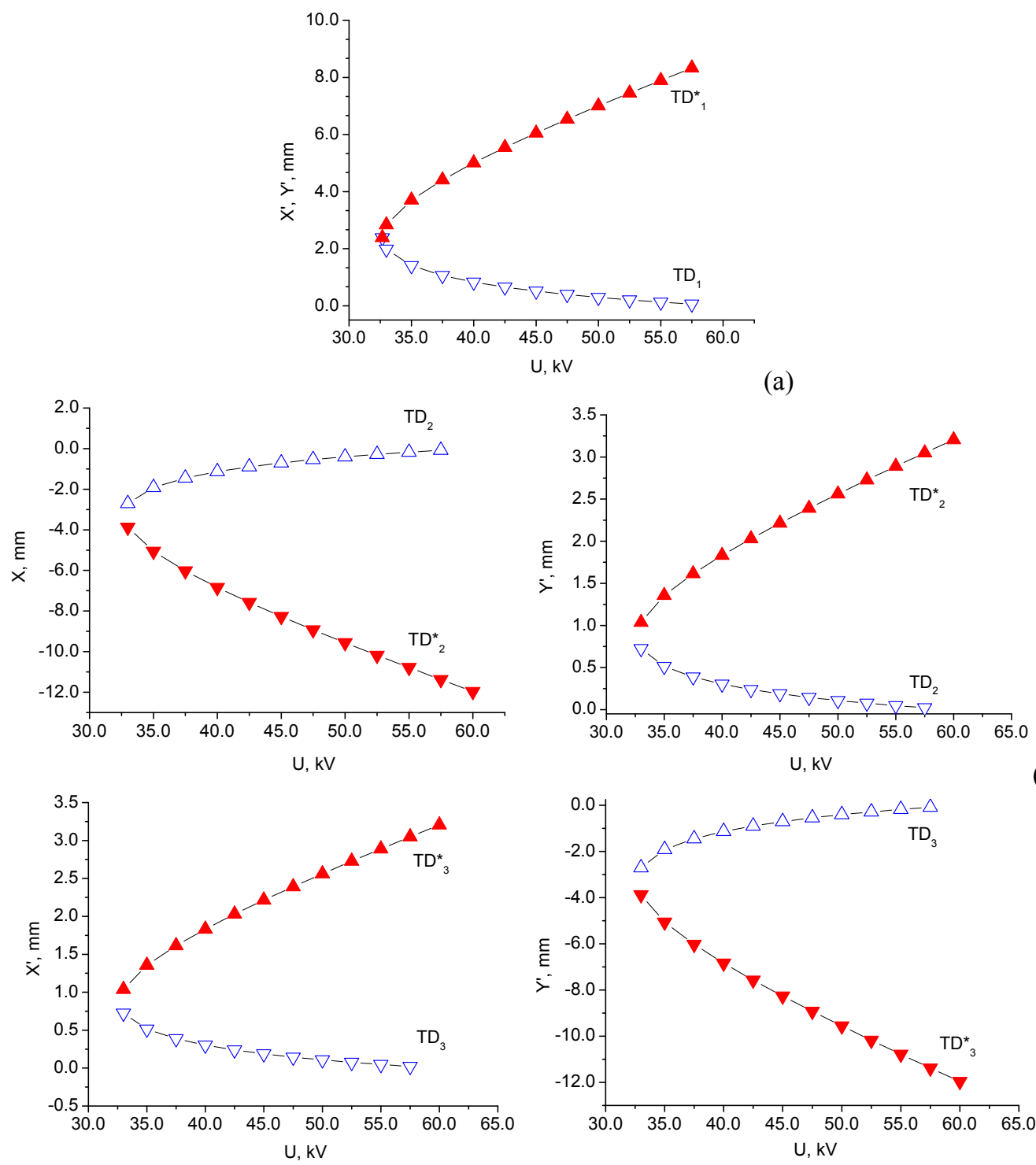

(b)

Fig. 5. Dependences of TDs coordinates on electric voltage for the crystals of symmetry group $\overline{4} 3 \mathrm{~m}:$ (a) TD and $\mathrm{TD}^{*}{ }_{1}$, (b) $\mathrm{TD}_{2}$ and $\mathrm{TD}^{*}{ }_{2}$, (c) $\mathrm{TD}_{3}$ and $\mathrm{TD}^{*}{ }_{3}$. 
For a hollow light beam with the radius of central dark spot greater than the radius at which $\mathrm{TD}_{1}, \mathrm{TD}_{2}$ and $\mathrm{TD}_{3}$ are located, one will deal with a doubly charged optical vortex, since the effective angle of optical indicatrix rotation changes its value by 360 deg whenever the tracing angle changes by the same value. When the beam radius gets smaller than the radius at which $\mathrm{TD}_{1}$, $\mathrm{TD}_{2}$ and $\mathrm{TD}_{3}$ are located, the singly charged optical vortex will be embedded in the beam centre, since the effective angle of optical indicatrix rotation at this radius changes by $180 \mathrm{deg}$ whenever the tracing angle changes by $360 \mathrm{deg}$. Notice that the total optical field of the outgoing beam contains seven singly charged optical vortices, the central one $\left(\mathrm{OV}_{0}\right)$ and six lateral ones $\left(\mathrm{OV}_{1}\right.$, $\mathrm{OV}_{1}^{*}, \mathrm{OV}_{2}, \mathrm{OV}_{2}^{*}, \mathrm{OV}_{3}$ and $\mathrm{OV}_{3}{ }_{3}$ ). Then one will deal with the crossover regime for the generation of optical vortices (see Ref. [15]): no optical vortex with a fractional charge will appear in this case.
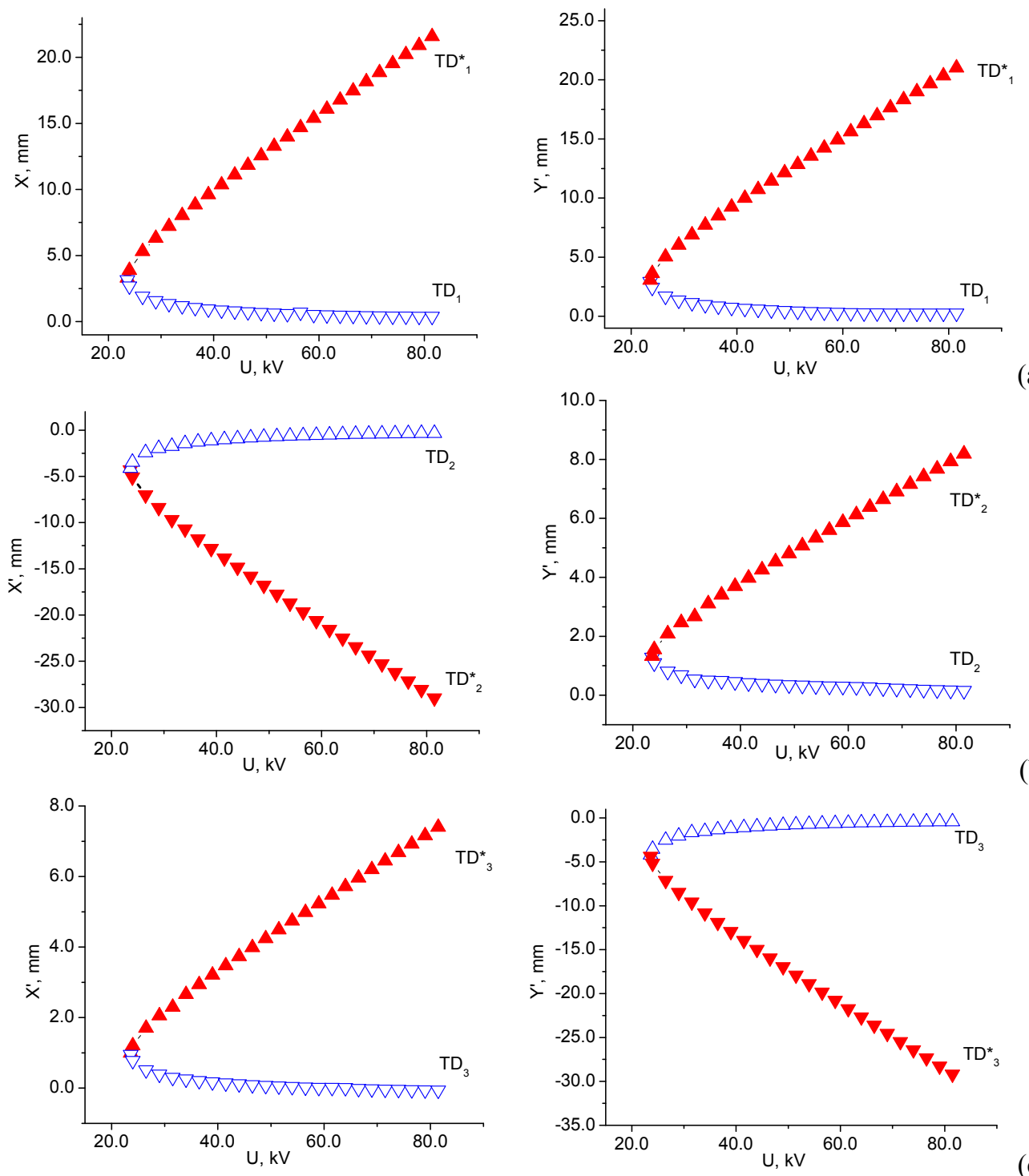

(b)

Fig. 6. Dependences of TDs coordinates on electric voltage for the crystals of symmetry group 23: (a) $T_{1}$ and $\mathrm{TD}^{*}{ }_{1}$, (b) $\mathrm{TD}_{2}$ and $\mathrm{TD}^{*}{ }_{2}$, (c) $\mathrm{TD}_{3}$ and $\mathrm{TD}^{*}{ }_{3}$. 
Now let us proceed to the crystals belonging to the point symmetry group 23 . In our simulations we use the following optical parameters that characterize such a crystal: $n=1.7$, $r_{11}=10^{-12} \mathrm{~m} / \mathrm{V}, R_{11}^{\prime}=1.925 \times 10^{-18} \mathrm{~m}^{2} / \mathrm{V}^{2}, R_{12}^{\prime}=0.875 \times 10^{-18} \mathrm{~m}^{2} / \mathrm{V}^{2}, R_{14}^{\prime}=0.021 \times 10^{-18} \mathrm{~m}^{2} / \mathrm{V}^{2}$, $R_{15}^{\prime}=-0.079 \times 10^{-18} \mathrm{~m}^{2} / \mathrm{V}^{2}, \quad R_{16}^{\prime}=-0.029 \times 10^{-18} \mathrm{~m}^{2} / \mathrm{V}^{2}, \quad$ and $\quad R_{66}^{\prime}=1.05 \times 10^{-18} \mathrm{~m}^{2} / \mathrm{V}^{2}$. The system of equations that defines the positions of the TDs becomes as follows:

$$
\left\{\begin{array}{l}
\left(E_{1}+E_{2}\right)\left(2 r_{11}^{\prime}+R_{16}^{\prime}\left(E_{1}-E_{2}\right)\right)+2 E_{3}\left(R_{15}^{\prime} E_{2}-R_{14}^{\prime} E_{1}\right)-2 R_{66}^{\prime} E_{1} E_{2}=0 \\
\left(E_{1}-E_{2}\right)\left(2 r_{11}^{\prime}+\left(R_{11}^{\prime}-R_{12}^{\prime}\right)\left(E_{1}+E_{2}\right)\right)+2 E_{3}\left(R_{14}^{\prime} E_{2}+R_{15}^{\prime} E_{1}\right)+2 R_{16}^{\prime} E_{1} E_{2}=0
\end{array} .\right.
$$

This system can be solved only numerically. As a result, we have obtained seven solutions, one of them being trivial $\left(X_{0}=0\right.$ and $\left.Y_{0}=0\right)$. The dependences of the coordinates for the other six defects on the electric voltage are presented in Fig.6. From Fig. 6 and Fig. 7 one can see that the behaviours of TDs in the crystals of the group 23 are similar to those observed earlier for the group $\overline{4} 3 \mathrm{~m}$.

Finally let us analyze the problem of sign of the charge of optical vortices produced by the TDs. When the vortices are generated with the aid of TDs of optical indicatrix orientation (as is the case with the q-plates), this sign is governed by the sign of spin angular momentum of the incident
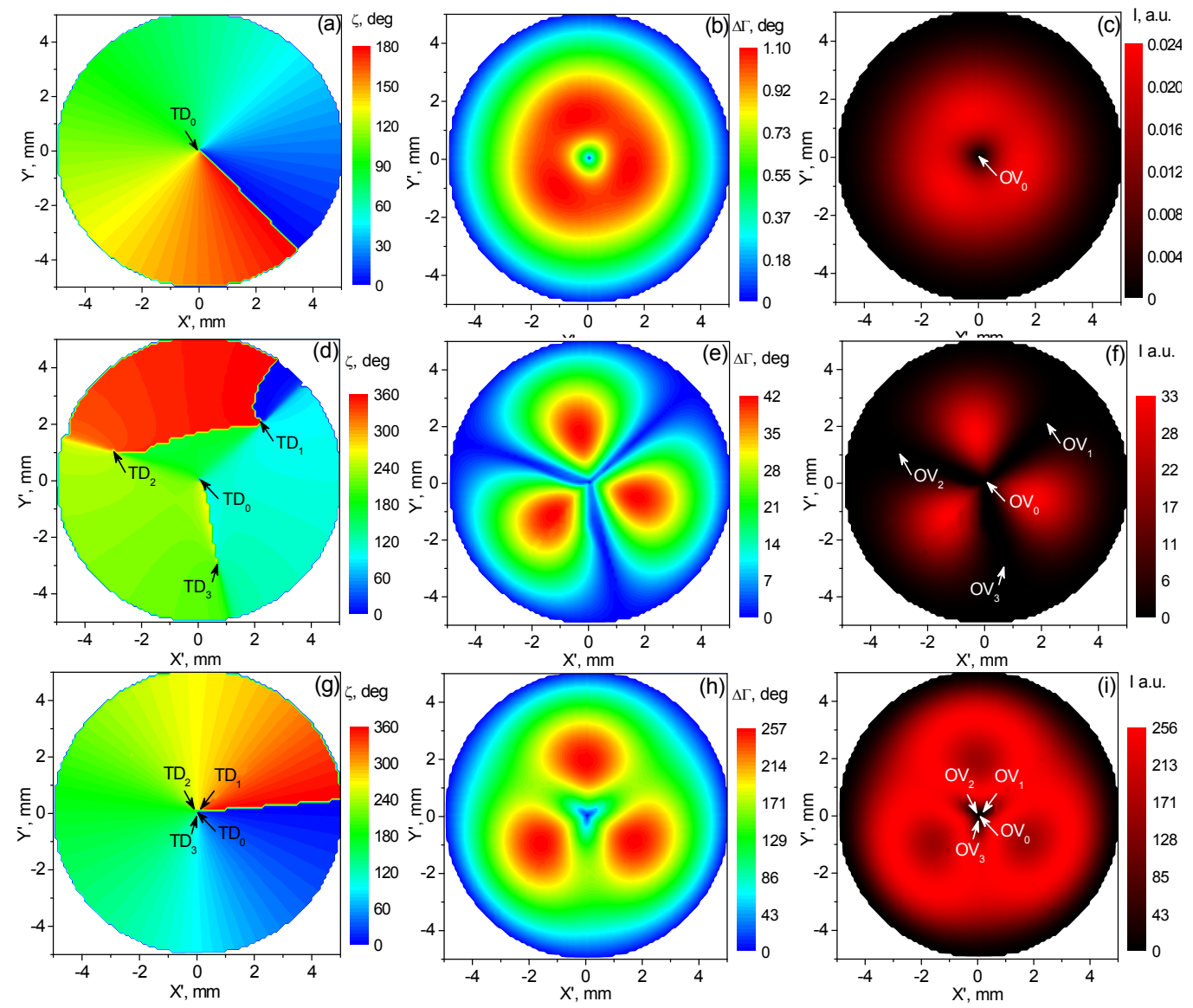

Fig. 7. Simulated spatial distributions for optical indicatrix rotation angle $(a, d, g)$, phase difference $(b, e, h)$ and light intensity behind the circular analyzer (c, f, i). The crystal belongs to the point group 23 , and the axes of the conical external field and the light beam are both parallel to [111]. The electric voltages are equal to 1 (a-c), 25 $(\mathrm{d}-\mathrm{f})$, and $80 \mathrm{kV}(\mathrm{g}-\mathrm{i})$. 
circularly polarized wave (see Ref. [16] and Eq. (5)). Namely, the sign of orbital angular momentum of the emergent vortex beam is the same as the sign of spin angular momentum of the incident wave. Here we are to notice that the sign of the vortex charge is not defined by the sign of the TD of optical indicatrix orientation. Thus, if a pair of TDs (e.g., $\mathrm{TD}_{1}$ and $\mathrm{TD}_{1}{ }_{1}$ ) is born, with the opposite signs of defect strengths characteristic for the individual defects within that pair, the relevant optical vortices would have the same sign, which is determined by the sign of the spin angular momentum of the incident light. Then the charge of the vortices is not conserved under topological reactions.

\section{Conclusions}

We have analyzed the behaviours of the TDs of optical indicatrix orientation under the conditions when the electrooptical Pockels and Kerr nonlinearities coexist in the crystals of cubic system, the conically shaped electric field is applied along the direction [111] in the crystal, and the light beam propagates in this direction. We have found that, under the aforementioned conditions, the behaviours of TDs in the crystals belonging to the point symmetry groups $\overline{4} 3 \mathrm{~m}$ and 23 turn out to be more complicated than that occurring in the hexagonal crystals of the symmetry group $\overline{6} 2 \mathrm{~m}$.

In particular, only four TDs are induced in the hexagonal crystals, whereas for the crystals of cubic system their quantity increases up to seven. Increase in the voltage gives birth to three pairs of TDs, conventionally referred to as $\mathrm{TD}_{1}$ and $\mathrm{TD}_{1}^{*}, \mathrm{TD}_{2}$ and $\mathrm{TD}_{2}{ }_{2}, \mathrm{TD}_{3}$ and $\mathrm{TD}_{3}^{*}$. These defects are characterized by half-integer values of their strengths and the opposite signs of strength within the pairs. With further increase in the voltage, the TDs drift apart. For example, the defect $\mathrm{TD}_{1}$ moves towards the centre of the beam cross-section, while $\mathrm{TD}^{*}{ }_{1}$ moves out of the beam aperture. We have also revealed that this process is reversible, i.e. the pairs of defects annihilate when the voltage decreases enough. The strength of the central TD does not change its value from $-1 / 2$ to 1 until the Pockels effect vanishes. Hence, the three TDs that move towards the central one never reach it with increasing voltage but only approach the central defect closely enough.

It has been found that the optical vortices produced by the TDs of optical indicatrix orientation are singly charged. The sign of their topological charge is defined by the sign of the spin angular momentum of the incident circular polarized wave rather than the sign of the strength of the corresponding TD of optical indicatrix orientation. This fact implies that the total charge of the optical vortices is not conserved under the topological reactions.

\section{References}

1. Volyar A, Shvedov V, Fadeyeva T, Desyatnikov A S, Neshev D N, Krolikowski W and Kivshar Yu S, 2006. Generation of single-charge optical vortices with an uniaxial crystal. Opt. Express. 14: 3724-3729.

2. Marrucci L, Manzo C and Paparo D, 2006. Optical spin-to-orbital angular momentum conversion in inhomogeneous anisotropic media. Phys. Rev. Lett. 96: 163905.

3. Piccirillo B, D'Ambrosio V, Slussarenko S, Marrucci L and Santamato E, 2010. Photon spinto-orbital angular momentum conversion via an electrically tunable q-plate. Appl. Phys. Lett. 97: 241104.

4. Groblacher S, Jennewein T, Viziri A, Weihs G and Zeillinger A, 2006. Experimental quantum cryptography with qutrits. New J. Phys. 8: 75.

5. Molina-Terriza G, Vaziri A, Rehácek J, Hradil Z and Zeilinger A, 2004. Triggered qutrits for quantum communication protocols. Phys. Rev. Lett. 92: 167903. 
6. David P. DiVincenzo, 1995. Quantum computation. Science. 270: 255-261.

7. Bouwmeester D, Pan J-W, Mattle K, Eibl M, Weinfurter H and Zeilinger A, 1997. Experimental quantum teleportation. Nature. 390: 575-579.

8. Grier D G, 2003. A revolution in optical manipulation. Nature. 424: 810-816.

9. Skab I, Vasylkiv Yu, Zapeka B, Savaryn V and Vlokh R, 2011. Appearance of singularities of optical fields under torsion of crystals containing threefold symmetry axes. J. Opt. Soc. Amer. A. 28: 1331-1340.

10. Skab I, Vasylkiv Yu, Savaryn V and Vlokh R, 2011. Optical anisotropy induced by torsion stresses in $\mathrm{LiNbO}_{3}$ crystals: appearance of an optical vortex. J. Opt. Soc. Amer. A. 28: 633640.

11. Skab I, Vasylkiv Yu and Vlokh R, 2012. Induction of optical vortex in the crystals subjected to bending stresses. Appl. Opt. 51: 5797-5805.

12. Skab I, Vasylkiv Yu, Smaga I and Vlokh R, 2011. Spin-to-orbital momentum conversion via electrooptic Pockels effect in crystals. Phys. Rev. A. 84: 043815.

13. Vasylkiv Yu, Krupych O, Skab I and Vlokh R. 2011. On the spin-to-orbit momentum conversion operated by electric field in optically active $\mathrm{Bi}_{12} \mathrm{GeO}_{20}$ crystals. Ukr. J. Phys. Opt. 12: $171-179$.

14. Vasylkiv Yu, Skab I and Vlokh R, 2014. Double-charged optical vortices generation on the basis of electrooptic Kerr effect. Appl. Opt. 53: B60-B73.

15. Vasylkiv Yu, Skab I and Vlokh R, 2014. Crossover regime of optical vortices generation via electro-optic nonlinearity: the problem of optical vortices with the fractional charge generated by crystals. J. Opt. Soc. Amer. A. 31: 1936-1945.

16. Marrucci L, 2008. Generation of helical modes of light by spin-to-orbital angular momentum conversion in inhomogeneous liquid crystals. Mol. Cryst. Liq. Cryst. 488: 148-162.

Vasylkiv Yu., Kryvyy T., Skab I. and Vlokh R. K. 2014. Behavior of topological defects of optical indicatrix orientation in cubic single crystals under conically distributed electric field. 1. The electric field and the optical beam parallel to the three-fold symmetry axis. Ukr.J.Phys. Opt. 15: $184-194$.

Анотація. У роботі досліджено поведінку топологічних дефектів (ТД) орієнтації оптичної індикатриси за умови співіснування покельсівської $і$ керрівської електрооптичних нелінійностей у кристалах кубічної сингонії, прикладання конічного електричного поля $i$ поширення пучка променів уздовж напрямку [111]. Виявлено, щзо зміна напруги приводить до народження трьох пар ТД, які характеризуються напівцілим значенням сили $і$ мають протилежні знаки цієї сили в межах пари. За умови зменшення напруги изі пари ТД анігілюють. Показано, щз топологічний заряд оптичних вихорів під час топологічних реакиій не зберігається. 